\title{
Helicon experiments and simulations in nonuniform magnetic field configurations
}

\author{
X. M. Guo, J. Scharer, Y. Mouzouris, and L. Louis \\ Department of Electrical and Computer Engineering, University of Wisconsin, Madison, Wisconsin 53706
}

(Received 26 January 1999; accepted 19 April 1999)

\begin{abstract}
Wave, antenna impedance, plasma density, and temperature anisotropy measurements are carried out for a helicon plasma source in nonuniform and uniform static magnetic fields. Strong axial density gradients associated with the nonuniform magnetic fields are observed to affect wave fields, absorption, and source efficiency. The wave field and antenna input impedance measurements are compared with a new simulation code which also calculates Poynting power flow and wave absorption profiles. Wave amplitude measurements are shown to decay more rapidly and the phase velocity varies over a wide range for the nonuniform static magnetic field case. (C) 1999 American
\end{abstract} Institute of Physics. [S1070-664X(99)00608-4]

\section{INTRODUCTION}

Helicon plasma sources have been investigated for their antenna coupling and wave mode properties as well as the mechanisms for efficient ionization, which has been achieved by researchers including Boswell et al., ${ }^{1}$ Komori et al., ${ }^{2}$ Shoji et al., ${ }^{3}$ and Chen et al. ${ }^{4}$ Recently, researchers including Molvik et al., ${ }^{5}$ Ellingboe et al., ${ }^{6}$ Degeling and Boswell, ${ }^{7}$ and Chen and Hershkowitz ${ }^{8}$ have examined fast electron production and Keiter et al. ${ }^{9}$ have examined helicon wave frequency dependent effects. These sources have a variety of applications including materials processing, ${ }^{10}$ a plasma source for magnetic fusion studies, ${ }^{11}$ sources for simulating space plasma interactions, ${ }^{9}$ laser plasma sources, ${ }^{12}$ and for space plasma thrustors. ${ }^{13}$

Helicon plasma sources in nonuniform magnetic fields have previously been investigated by researchers including Boswell et al. ${ }^{14}$ Chen et al.,${ }^{15,16}$ and Gilland et al. ${ }^{17}$ The primary observation has been an increase in the plasma density when a cusp or nonuniform magnetic field has been placed in the vicinity of the helicon antenna. The reasons for this increased plasma density have not been examined in detail but fast electrons and improved confinement are mentioned as possible contributors to the observation. We have carried out experiments and measurements on the effects of nonuniform magnetic fields on the source operation including axial and radial density profiles, the position and role of the electron cyclotron resonance $\left(\omega=\omega_{c e}\right)$, the gradient in the magnetic field, the antenna input impedance, the wave magnetic field variation and wave number spectrum, and the transverse and longitudinal electron temperatures. In addition to this, we have utilized a new two-dimensional (2-D) $(r, z)$ finitedifference, full-wave, helicon simulation code we developed, MAXEB,${ }^{18,19}$ to compare with the experimental results. This research has been carried out to explore the physics involved and enhanced ionization efficiency occurring in nonuniform magnetic fields for efficient plasma production.

This paper is organized as follows. A description of the experimental configuration and diagnostics is presented in Sec. II. The experimental measurements and the corresponding simulations are discussed in Secs. III and IV, respec- tively. In Sec. V, we discuss plasma confinement and linear and nonlinear wave processes in interpreting the observations and correspondence with the simulations.

\section{EXPERIMENTAL APPARATUS AND DIAGNOSTICS}

\section{A. Plasma source}

The radio-frequency helicon plasma source facility is schematically shown in Fig. 1. The plasma chamber is made of 10 -cm-diam by $122-\mathrm{cm}$-length cylindrical $\mathrm{Pyrex}^{\mathrm{TM}}$ pipe and is pumped to a base pressure of $3 \times 10^{-7}$ Torr by a 550 $\ell / \mathrm{s}$ turbomolecular pump. Diagnostic probes can be inserted through radial ports on the Pyrex pipe and end plate flanges. A nude Bayard-Alpert ionization and a Baratron pressure gauge are used to measure low (less than $10^{-4}$ Torr) and high (above 1 mTorr) pressures, respectively, through a gauge controller.

A $13.56 \mathrm{MHz}, 1.4 \mathrm{~kW}$ rf power amplifier is connected to a Nagoya type III (15-cm-long) antenna through a matching network to couple rf power to the plasma. In order to provide long probe lifetime in a Pyrex glass chamber, the radiofrequency source is operated in a pulsed mode with a pulse width of $8 \mathrm{~ms}$ and $10 \%$ duty cycle. The rise time of the $\mathrm{rf}$ power is $15 \mu$ s and the fall time is $3 \mu \mathrm{s}$. Working gases, such as argon and nitrogen, are fed into the chamber via flow controllers and a gas mixer through a radial port. A static magnetic field $\left(B_{0}\right)$ of up to $1.5 \mathrm{kG}$ is produced by a set of solenoid coils $7 \mathrm{~cm}$ thick with inner and outer diameters of 20 and $50 \mathrm{~cm}$, respectively. We have examined two types of axial magnetic field configurations, i.e., a uniform magnetic field and nonuniform magnetic field with a strong gradient near one end of the coupling antenna, as shown in Fig. 2. By utilizing two magnet power supplies the value of the minimum magnetic field near the left end of the antenna can be adjusted to include or eliminate the electron cyclotron resonance $\left(\omega=\left|\omega_{c e}\right|\right.$ at $\left.4.8 \mathrm{G}\right)$.

\section{B. Diagnostic probes}

\section{Langmuir probes}

A flat tantalum disk of diameter $2.4 \mathrm{~mm}$ was employed to measure the ion saturation current with a fixed bias $(-70$ 


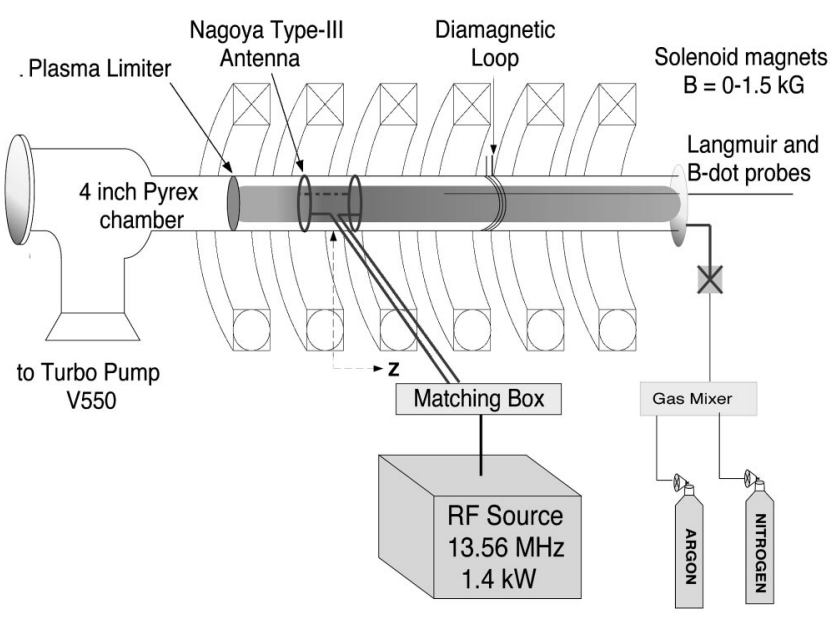

FIG. 1. Helicon experimental facility.

V). It is oriented with its flat side parallel to the magnetic field so that electron and ion currents can flow along field lines to the probe collection areas. ${ }^{1}$ The electron temperature was obtained by sweeping either single or double probes. The plasma density is obtained from the ion saturation current and the electron temperature, as given by the expression: $I_{\mathrm{sat}}=0.6 e n A\left(\kappa T_{e} / M\right)^{1 / 2}$, where $n$ is the plasma density, $T_{e}(\mathrm{eV})$ is the electron temperature, $\kappa$ is the Boltzmann constant, $A$ is the probe area, and $M$ is the ion mass. The parallel temperature was measured with a single-sided (dielectric coating on the backside) 1-mm-diam disk probe oriented perpendicular to the magnetic field.

Single and double cylindrical probes were also employed, which were made of tungsten wires with a diameter of $0.2 \mathrm{~mm}$, a length of $5 \mathrm{~mm}$, and a distance of $1.5 \mathrm{~mm}$ between them. They were oriented parallel to $B_{0}$ to measure the perpendicular component of electron temperature. Research by Aikawa, ${ }^{20}$ Amemiya et al. ${ }^{21}$ Himmel et al.,${ }^{22}$ and Tichy et al. ${ }^{23}$ have verified the use of these probe methods for perpendicular and parallel electron temperature measurements in high density electron cyclotron resonance plasmas.

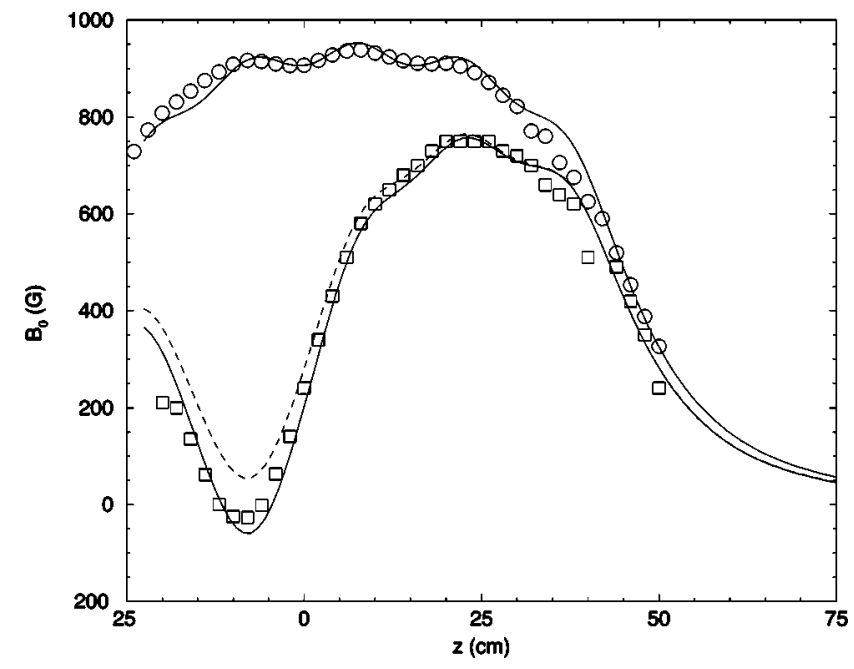

FIG. 2. Uniform and nonuniform magnetic field configurations. $B_{\min }=50 \mathrm{G}$ $(--)$; theoretical calculations $(-)$; and measurements $(\bigcirc$ and $\diamond)$.
The electron temperature was deduced from the slope of semilog of the $I-V$ curves starting from the tangent to the ion saturation current where magnetic field distortions can be expected to be less severe. Only those electrons with large gyroradii outside the sheath $(<15 \mu \mathrm{m})$ are then collected. ${ }^{24}$ Radio-frequency chokes at $13.56,27.12$, and $40.68 \mathrm{MHz}$ were used to reduce distortion of the $I-V$ curves. The $I-V$ characteristic curves are obtained by a Hewlett-Packard floating voltage sweeper ( -60 to $60 \mathrm{~V}, \tau=2 \mathrm{~ms}$ ) and a $50 \Omega$ resistor. The $I-V$ curves are recorded by a LeCroy 9314AM oscilloscope and sent to the computer controlled by a LABVIEW program.

\section{Radio-frequency magnetic probe}

A balanced magnetic probe was used to measure the magnitude and phase variations of axial wave component $B_{z}$ in the radial and axial directions. The probe consists of a center-tapped six-turn coil with a diameter of $2 \mathrm{~mm}$, and it is axially oriented parallel to $B_{0}$ in a $1 / 4$ in. stainless steel tube with two $2 \mathrm{~mm} \times 5 \mathrm{~mm}$ slits to allow wave magnetic field pickup. The slits are covered with a Pyrex glass tube to isolate the coils from direct plasma contact. A radio-frequency BALUN circuit is used to effectively reject electrostatic capacitive pickup. ${ }^{25}$ For wave phase measurements, the input antenna current measured by a Rogowski coil is used as reference signal for the mixer.

Calibration of the helicon wave $B_{z}$ field measurement is carried out utilizing a 45-turn solenoid coil $(3.8 \mathrm{~cm}$ wide $\times 11.8 \mathrm{~cm}$ diam) driven at $13.56 \mathrm{MHz}$. The coil current was measured by a Rogowski coil. The wave probe is placed at the center of the coil and the relationship between the wave magnetic field, $B_{z}$, and induced voltage is calibrated.

\section{Diamagnetic loop}

The diamagnetic loop is made of a semirigid coaxial cable, with the center conductor connected to the outer conductor at the end of the third axial loop. It is located on the outer surface of the Pyrex tube at $z=17 \mathrm{~cm}$, which is near the center of the helicon plasma. The diamagnetic loop signal is integrated by an $R C$ integrator with a $1 \mathrm{~ms}$ time constant and averaged over 150-200 shots to reduce noise. In this study, the diamagnetic loop signals for the plasma decay after the rf power is turned off were used to obtain the steady-state electron temperature. Given the plasma density and its radial profile, the electron temperature component perpendicular to the magnetic field can be deduced when the plasma is axially symmetric and sufficiently long. ${ }^{22,26}$

\section{EXPERIMENTAL OBSERVATIONS}

The experimental observations examined the radiofrequency matching of the antenna and its impedance utilizing network analyzer techniques. The axial and radial profiles of plasma density as well as transverse and longitudinal electron temperature were obtained utilizing single and double Langmuir probes and a diamagnetic loop. A comparison of the uniform and nonuniform magnetic field configurations was obtained for the same input power levels of 1.1 $\mathrm{kW}$ at $13.56 \mathrm{MHz}$. Steady-state reflections and losses in the 


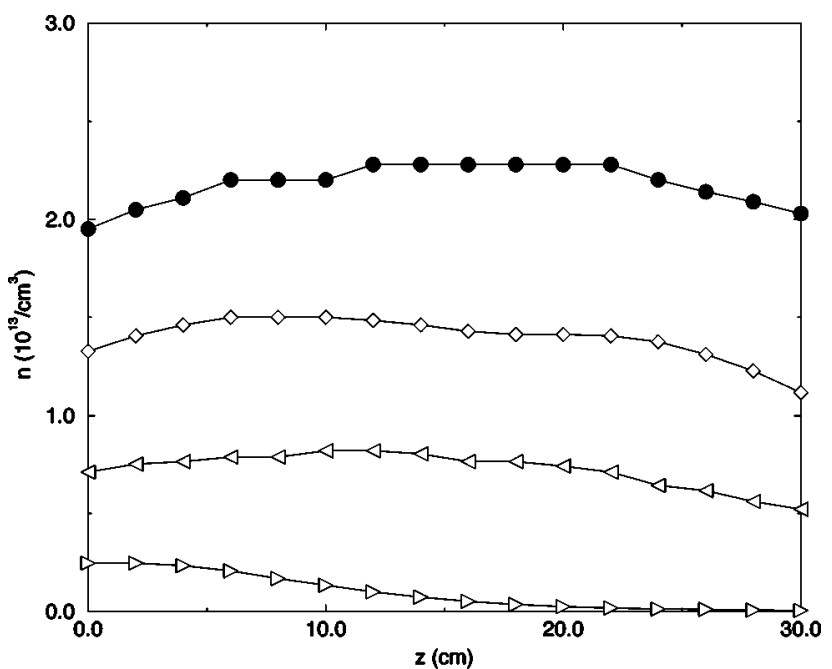

FIG. 3. Plasma axial density profile with uniform magnetic field in $\mathrm{Ar}$ at 1 mTorr pressure and $1.1 \mathrm{~kW}$ rf power. $B_{0}=0(\triangleright), B_{0}=640 \mathrm{G}(\triangleleft), B_{0}$ $=840 \mathrm{G}(\diamond)$, and $B_{0}=1120 \mathrm{G}(\bullet)$.

matching circuit were measured to be less than $2 \%$. The plasma density at a $1 \mathrm{mT}$ Torr filling pressure for argon as a function of the applied uniform magnetic field is shown in Fig. 3. Note that the plasma density measured at $t=5 \mathrm{~ms}$ in the steady state after the initiation of the rf pulse rises as the magnetic field is increased and is $1-2 \times 10^{13} \mathrm{~cm}^{-3}$ when the magnetic field is increased to the $840-1120 \mathrm{G}$ range. The corresponding electron temperatures when the magnetic field is $840 \mathrm{G}$ are shown in Fig. 4. The electron temperatures are in the range of $3.6 \mathrm{eV}$ for the transverse temperature and 2.9 $\mathrm{eV}$ for the parallel temperature for the uniform case.

The nonuniform magnetic field case, with a $750 \mathrm{G}$ peak magnetic field, the same coupled power level at the same filling pressure of $1 \mathrm{mTorr}$ provides a plasma density profile as shown in Fig. 5. The strong magnetic field gradient in the source region (see Fig. 2) yields a density profile which has a substantial gradient rising from $2 \times 10^{12} \mathrm{~cm}^{-3}$ at the left

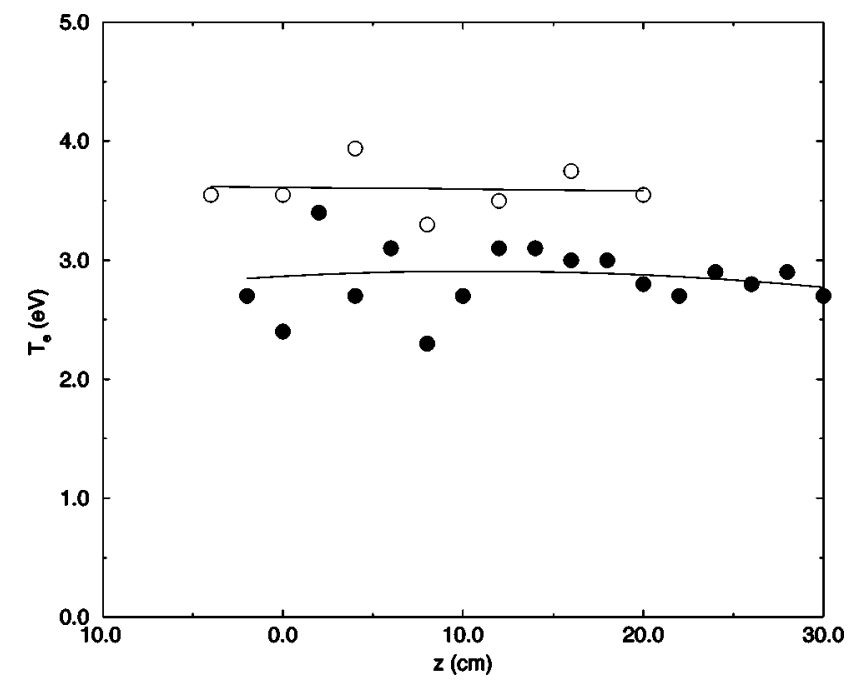

FIG. 4. Electron temperature for a uniform magnetic field in Ar at $1 \mathrm{mTorr}$ pressure, $1.1 \mathrm{~kW}$ rf power: parallel electron temperature $(\bullet)$, transverse electron temperature measured by a double probe $(\bigcirc)$.

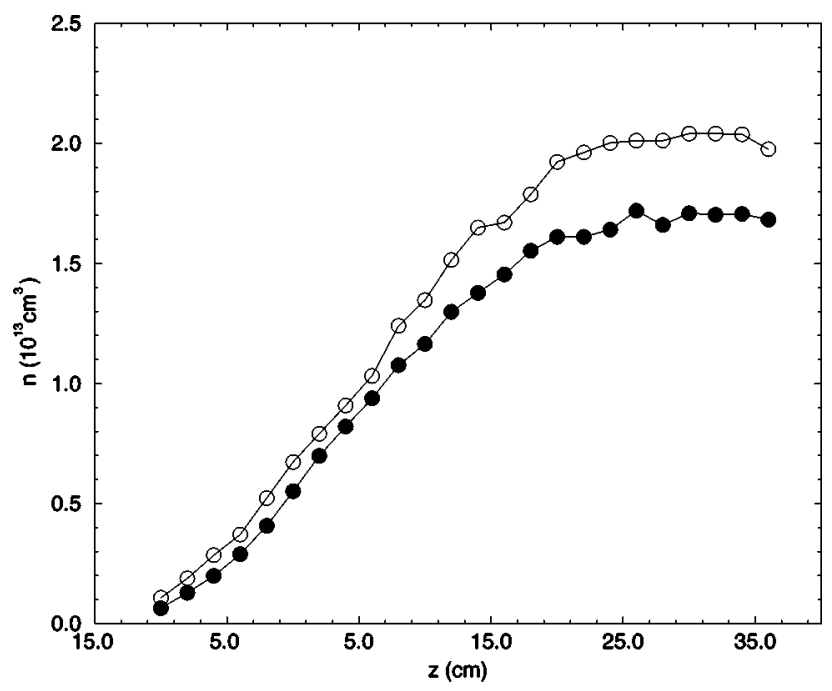

FIG. 5. Plasma axial density profiles for nonuniform magnetic field in Ar at $1 \mathrm{mT}$ Torr pressure and $\mathrm{rf}$ power level $1.1 \mathrm{~kW}$. The minimum $B_{0}$ values around $z=-7.5 \mathrm{~cm}$ are $-10 \mathrm{G}(\bullet)$ and $50 \mathrm{G}(\bigcirc)$.

end of the type-III antenna at $z=-7.5 \mathrm{~cm}$ to $2.0 \times 10^{13} \mathrm{~cm}^{-3}$ at $z=30 \mathrm{~cm}$. Note that the density profiles near the "resonance" portion at $z=-4 \mathrm{~cm}$ are quite close to those when the local minimum in the magnetic field is increased to $50 \mathrm{G}$ and the "resonance" is removed. The plasma density has a slight flattening in the local mirror near the resonance on the left-hand side of the antenna and rapidly decreases as one moves further away from the antenna. The radial density profile is broader $(+20 \%)$ when compared to the uniform magnetic field case at $z=15 \mathrm{~cm}$. The corresponding transverse and parallel electron temperatures were measured by single and disk Langmuir probes and a diamagnetic loop which ranges from 3.5 to $7 \mathrm{eV}$ and from 3 to $5 \mathrm{eV}$ over a wide range of axial positions are shown in Fig. 6. An error bar indicating the range of transverse temperature measurements is shown for the position $z=17 \mathrm{~cm}$. The radial variation of electron temperature has a broad radial profile.

We carried out detailed measurements of the effect of the magnetic gradient and the role of the electron cyclotron resonance in the plasma source operation for the nonuniform magnetic field. We accomplished this by driving one coil, the one centered at $z=-7.5 \mathrm{~cm}$ independent of the others and can vary the depth of the magnetic field minimum as shown in Fig. 2. The plasma has a very sharp magnetic gradient and a substantial density gradient near the vacuum cyclotron resonance which occurs at $\pm 4.8 \mathrm{G}$ for the operating frequency. Also, the plasma beta is quite substantial near the minimum magnetic field which increases the magnetic field gradient near the resonance. We carried out measurements which increased the minimum magnetic field near the resonance zone to $50 \mathrm{G}$ and found that the source operated in a similar manner to the case in which resonance is present, with comparable density and temperature profiles as shown in Figs. 5 and 6 . The steady-state $(\tau=5 \mathrm{~ms})$ electron temperatures are the same when the resonance is included or when it is removed by increasing the minimum magnetic field to $50 \mathrm{G}$. Thus relatively small effects are caused by the 


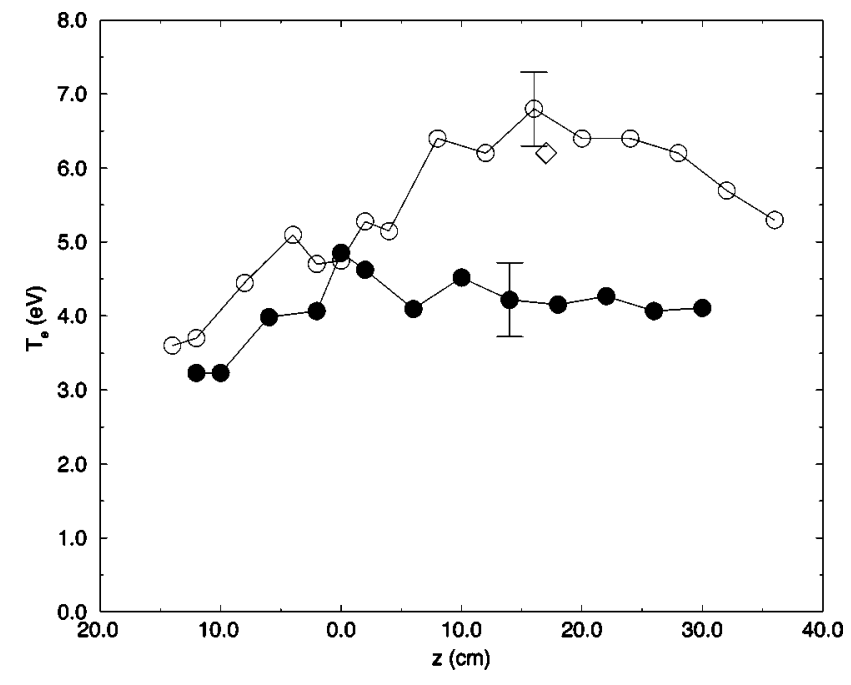

FIG. 6. Electron temperature for a nonuniform magnetic field in $\mathrm{Ar}$ at 1 mTorr pressure and for $11.1 \mathrm{~kW}$ coupled rf power. Parallel electron temperature $(\bullet)$; transverse electron temperature from a double probe $(\bigcirc)$; and transverse electron temperature from diamagnetic loop $(\diamond)$.

existence of the cyclotron resonance near the antenna region in the steady-state condition of the plasma source. This is because the strong magnetic gradient and wave $B_{z}$ values comparable to the resonant static magnetic field amplitude destroy the wave-particle coherence over a gyro-orbit. In addition, the strong plasma density and magnetic field gradients reflect the wave power from this region.

The steady-state transverse electron temperature is $1.5-2$ $\mathrm{eV}$ above the parallel temperature except well under the antenna region from $z=0$ to $-10 \mathrm{~cm}$. The increase in the transverse temperature as one moves toward the higher magnetic field region can be caused by the increase in magnetic compression although additional collisional helicon mode heating is occurring in the intervening region.

A twofold increase in the total volume integrated kinetic energy density is noted for the nonuniform magnetic field configuration when compared to the uniform magnetic field case for the same power input. This can be attributed to more efficient plasma ionization for the coupled helicon modes and improved confinement of the plasma formed for the good curvature magnetic field case. Note also that the volume-average beta for the nonuniform case is improved by a factor of 4 when compared with the uniform case.

The "blue mode" 1,6 operation of the source caused by intense radiation from the argon II state is quite intense in the core for the nonuniform magnetic field from $z=-3 \mathrm{~cm}$ to $z=55 \mathrm{~cm}$. The blue mode occurs in the inner $4 \mathrm{~cm}$ diameter of the source and is identified with the production of fast electrons which can excite the argon II state by either $15 \mathrm{eV}$ energy from the ground ion state or $30 \mathrm{eV}$ for the ground atomic state of argon. For the uniform magnetic field case at the same power input, the "blue mode" is $3 \mathrm{~cm}$ in diameter and less intense than for the nonuniform magnetic field case.

We carried out higher pressure nitrogen gas experiments to determine the source efficiency for this regime. We found that the nonuniform magnetic field at higher peak values was much more efficient than the uniform case at higher nitrogen

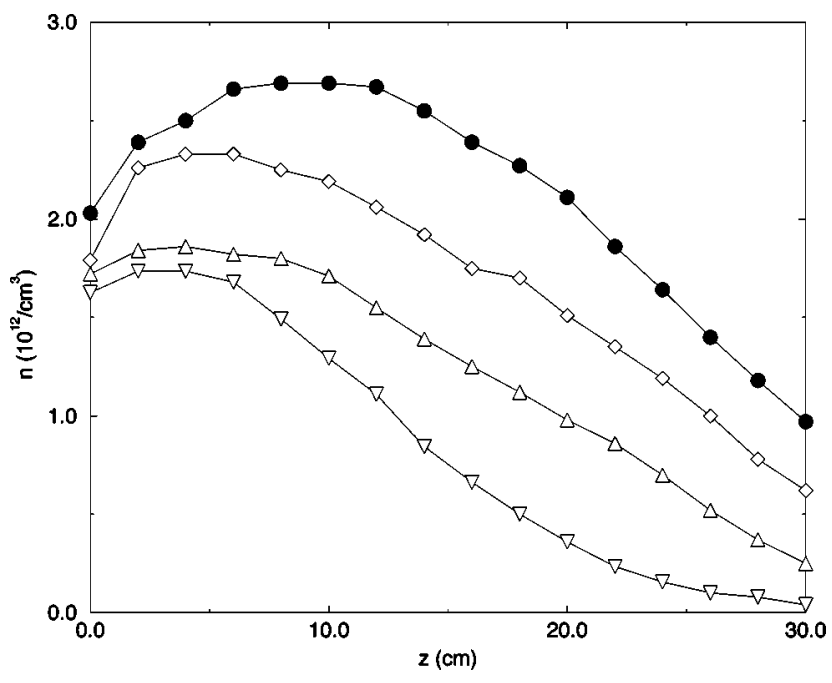

FIG. 7. Plasma axial density profiles for a nonuniform magnetic field in $N_{2}$ at 100 mTorr pressure and $1.1 \mathrm{~kW}$ rf power levels. $B_{0}=250 \mathrm{G}(\triangleright), B_{0}$ $=500 \mathrm{G}(\triangleleft), B_{0}=750 \mathrm{G}(\diamond)$, and $B_{0}=1000 \mathrm{G}(\bullet)$.

pressures. The nitrogen plasma axial density profile at 100 mTorr and $1.1 \mathrm{~kW}$ of coupled power is shown in Fig. 7. The peak density for nitrogen at higher magnetic fields is 2 $\times 10^{12} \mathrm{~cm}^{-3}$ and has a similar axial density gradient as is the case for argon. The maximum electron temperature was $6 \mathrm{eV}$ for this high pressure case and its axial variation is shown in Fig. 8. The electron temperature is expected to be more isotropic for this higher pressure and larger electron-neutral collision cross section. Higher magnetic field intensities also assisted in efficiently forming the plasma for this case. For this higher pressure with a uniform magnetic field, it was not possible to initiate and sustain the nitrogen plasma at the same power levels with the rf pulse alone.

The impedance of the Nagoya type-III antenna is measured using a Hewlett-Packard 8753A RF Network Analyzer for both the uniform and nonuniform magnetic field configurations. The conjugate match principle is utilized. Once the matching circuit is tuned to reduce the reflections

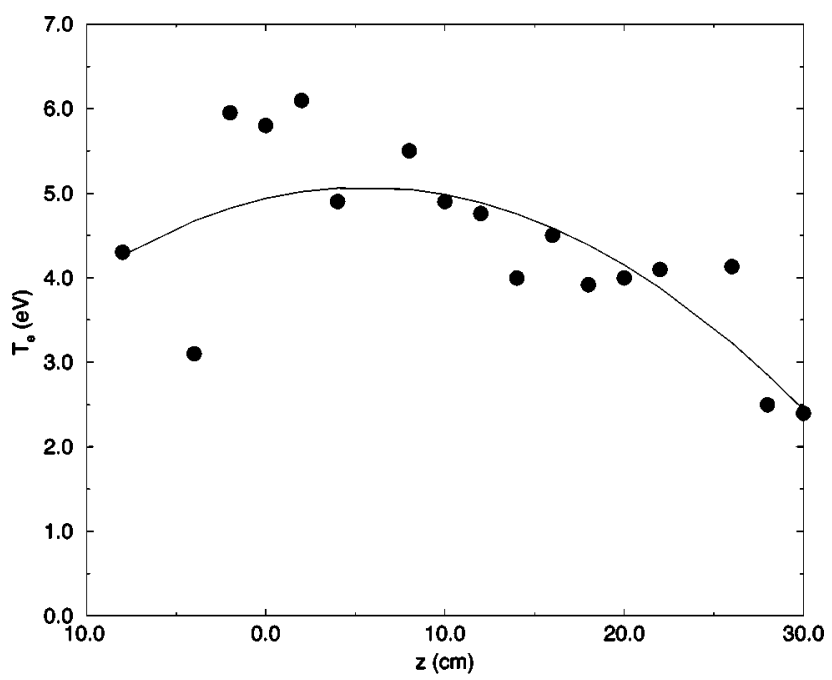

FIG. 8. Electron temperature for nonuniform magnetic field of $B_{0}=1000 \mathrm{G}$ in $N_{2}$ at 100 mTorr pressure and $1.1 \mathrm{~kW}$ rf coupled power. 
to a minimum, the antenna couples the rf power efficiently to the plasma. The antenna connection port of the two capacitor matching circuit is connected to the network analyzer and the conjugate impedance of the matching circuit, $R_{m}-j X_{m}$, is measured. Time domain reflectometry (TDR) is used to find the electrical length of the transmission line connecting the matching network to the antenna. The impedance, $R_{m}$ $-j X_{m}$ is rotated on the Smith chart toward the antenna to obtain the impedance $R-j X$ at the terminals of the Nagoya type-III antenna. Since this is the conjugate match impedance for minimum reflection, the impedance of the antenna will be $R+j X$.

For the nonuniform magnetic field configuration, the impedance of the antenna is measured to be $0.92+j 11 \Omega$ at 13.56 MHz. This is for an input power of $1.1 \mathrm{~kW}$ with a reflected power of $11 \mathrm{~W}$ at a pressure of $1 \mathrm{mTorr}$. The antenna impedance is measured to be the same value even when the input power is reduced to $500 \mathrm{~W}$. The blue core mode exists for these input power levels. But when the input power is reduced to $200 \mathrm{~W}$ and the matching network is retuned to provide a minimum reflection of $5 \mathrm{~W}$, the blue core does not exist. The antenna impedance is then measured to be $0.78+j 12.5 \Omega$. The lack of blue core mode reduces the antenna coupling efficiency and reduces the resistive part of impedance and antenna $Q$. For the uniform magnetic field case we obtain a somewhat lower antenna impedance of $0.85+j 10.4 \Omega$ by the same method at a $1.1 \mathrm{~kW}$ coupled power level.

To further examine the mechanisms which occur for the nonuniform magnetic field case we carried out wave $B_{z}$ measurements and determined their variation in the plasma. We carried out radio-frequency mixer measurements of the wave phase at $13.56 \mathrm{MHz}$. Figure 9 illustrates the amplitude and phase of the $B_{z}$ wave field at different radii. The wave $B_{z}$ field for the uniform case, shown in Fig. 10, has a much lower amplitude. For the nonuniform case, there is a strong gradient in $B_{z}$ as one moves away from the antenna and it is of much larger amplitude under the antenna extending well to the right away from the antenna near $z=40 \mathrm{~cm}$. The strong decay of the wave $B_{z}$ amplitude suggests strong coupling of rf power to the plasma in the nonuniform magnetic field configuration. It is noted that the local phase velocity for the nonuniform magnetic field case under the source region at $r=0.93 \mathrm{~cm}$ for $z \leqslant 0 \mathrm{~cm}$ corresponds to resonant electron energies $\left(E_{e}=m_{e} \mathrm{v}_{\phi}^{2} / 2\right)$ as low as $5 \mathrm{eV}$, while it gradually increases to $38 \mathrm{eV}$ for $z \geqslant 5 \mathrm{~cm}$. The strong decay of wave amplitude and the increase in phase velocity for $z$ $\geqslant 5 \mathrm{~cm}$ indicates possible electron heating and acceleration. This gradual increase from very low trapped electron velocities $(5 \mathrm{eV})$ to higher phase velocities as the wave moves away from the antenna region can be a very effective acceleration mechanism. The electron energy corresponding to the phase velocity of the wave for the uniform case (Fig. 10) has a higher range of local phase velocities as one moves away from the antenna and corresponds and varies from 25 to 40 $\mathrm{eV}$ for $r=0.93 \mathrm{~cm}$.

It should also be noted that the nonuniform magnetic field configuration produces a more intense and large axial and radial extent "blue core" which is indicative of the ex-
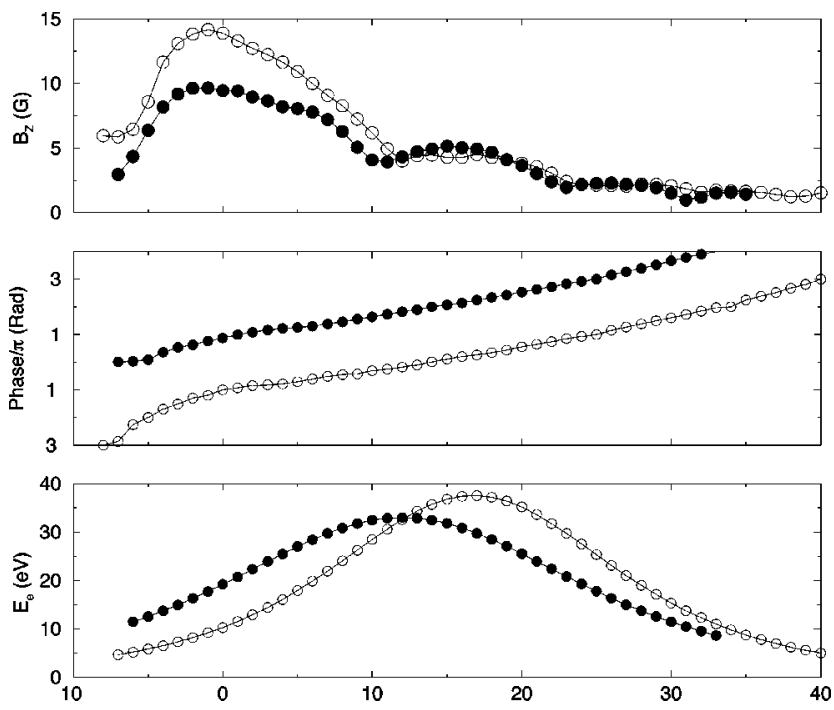

FIG. 9. Wave $B_{z}$ amplitude, phase, and resonant electron energy $\left(E_{e}\right)$ for the nonuniform magnetic field case at radial positions $0.93 \mathrm{~cm}(\bigcirc)$ and 1.54 $\mathrm{cm}(\bullet)$. The 15-cm-long Nagoya type-III antenna is centered at $z=0$.

citation of the argon II state which requires $15-30 \mathrm{eV}$ electrons to excite it from the ground argon ion or atomic states. In addition, the "blue core" plasma does not follow the flux compression that the magnetic field lines exhibit as one moves from the antenna toward larger axial position, which has been noted earlier by Boswell. ${ }^{1}$ This can be due to the strong radio-frequency wave fields which transport fast electrons axially without following the static magnetic field lines. The radial density profile as measured by Langmuir probes is broader $(20 \%)$ for the nonuniform magnetic field case as shown in Fig. 11.

\section{COMPUTER SIMULATION OF EXPERIMENTAL DATA}

We have developed MAXEB, ${ }^{18,19}$ a new 2-D $(r, z)$ cylindrical plasma wave code, which can treat both axial and
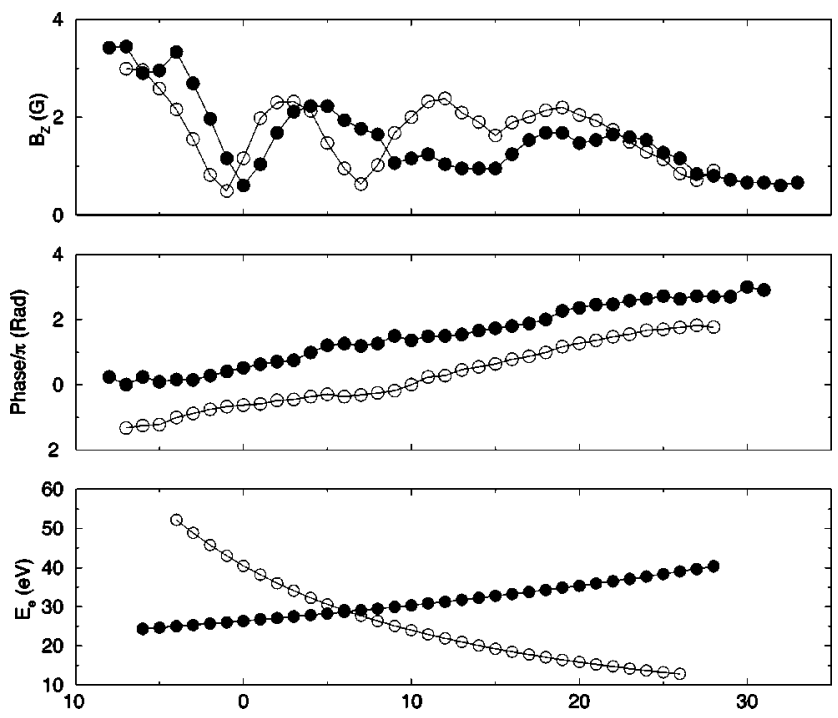

FIG. 10. Wave $B_{z}$ amplitude, phase, and resonant electron energy ( $\left.E_{e}\right)$ for the uniform magnetic field case at radial positions $0.93 \mathrm{~cm}(O)$ and $0.63 \mathrm{~cm}$ (-). The 15-cm-long Nagoya type-III antenna is centered at $z=0$. 


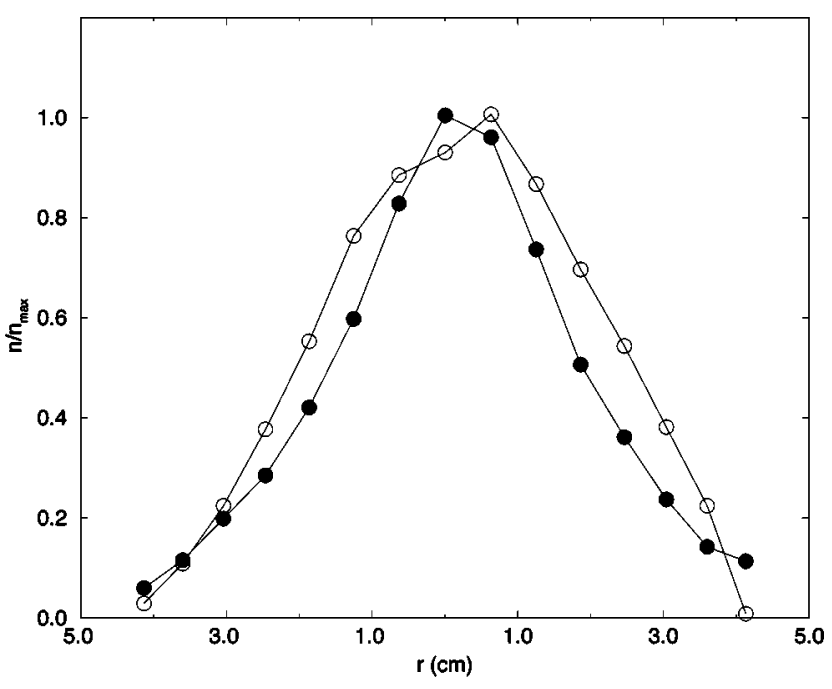

FIG. 11. Normalized plasma radial density profiles at $1 \mathrm{mTorr}$ pressure, 1.1 $\mathrm{kW}$ rf power at position $z=15 \mathrm{~cm}$. Uniform magnetic field $B_{0}=840 \mathrm{G}(\bigcirc)$, nonuniform magnetic field $B_{0}=750 \mathrm{G}(O)$. The $15-\mathrm{cm}-$ long antenna is centered at $z=0$.

radial variations in the plasma density and magnetic field. The code calculates the three-dimensional (3-D) electromagnetic wave fields and collisionless Landau as well as collisional linearized power absorption in an inhomogeneous, warm plasma. The cylindrical magnetized plasma is surrounded by a $\mathrm{rf}$ inductive coil and both are enclosed in a metal conducting tube. The code is written for a general orthogonal curvilinear coordinate system $\left(u_{1}, u_{2}, u_{3}\right)$, but for the purpose of this paper cylindrical coordinates are used. MAXEB assumes axisymmetry and Fourier analysis in the azimuthal direction to reduce the three-dimensional boundaryvalue problem to two dimensions: $u_{1}$ and $u_{3}$, or in cylindrical coordinates, the radial $(r)$ and axial $(z)$ direction, respectively. The azimuthal $(\phi)$ coordinate is labeled by $u_{2}$. The fields can be then decomposed as follows:

$$
[\mathbf{E}, \mathbf{H}]=\sum_{m=-\infty}^{m=+\infty}\left[\mathbf{E}, \mathbf{H}\left(u_{1}, u_{3}\right)\right] e^{i m u_{2}} .
$$

A typical run using a grid size of $50 \times 120$ in the radial and axial positions for a cylindrical plasma column of length $L$ $=120 \mathrm{~cm}$ and radius $r=5 \mathrm{~cm}$ and utilizing only the dominant azimuthal $m=+1$ mode requires about 5 min of run time on our Sun Ultra2 model 2300 workstation.

We have utilized this code to compare the plasma input impedance, wave $B_{z}$ fields, and to obtain the Poynting vector and wave absorption for the experimentally measured uniform and nonuniform density and magnetic field configurations. Figure 12 illustrates the Poynting vector power flow and contours of increased absorption for the nonuniform magnetic field case corresponding to Fig. 2 with the density profiles as obtained from Fig. 5 and the corresponding radial profiles. The minimum magnetic field near $z^{\prime}=30 \mathrm{~cm}\left(z^{\prime}\right.$ $=z+37.5 \mathrm{~cm}$ ) was set to $60 \mathrm{G}$. For this case, $26 \%$ of the wave power is collisionally absorbed in the region $z^{\prime}$ $=28-32 \mathrm{~cm}$ and $74 \%$ of the wave power is absorbed in the remaining region.

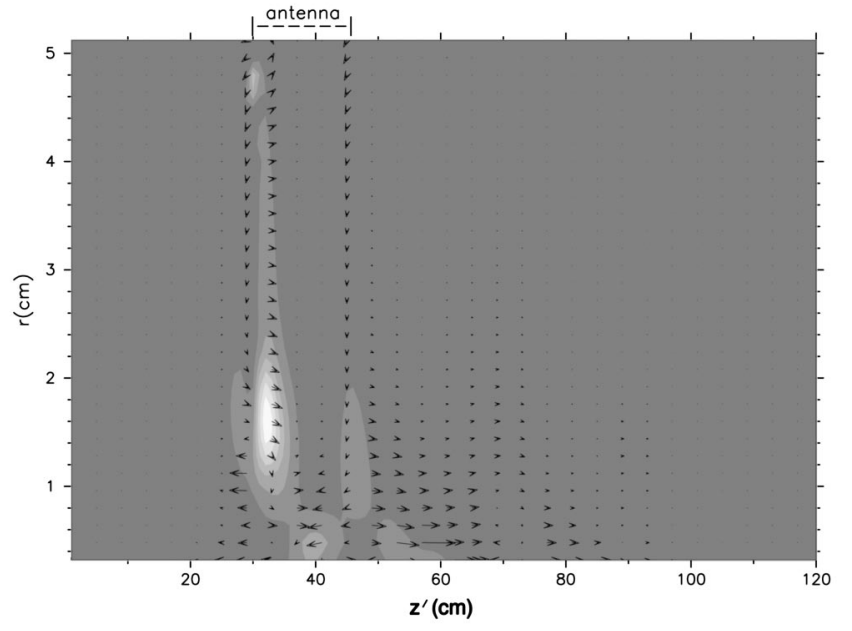

FIG. 12. Power flow and power absorbed $\left[\max \left(P_{\text {abs }}\right)=7.2 \mathrm{~W} / \mathrm{cm}^{3}\right)$ by the electrons for the experimentally measured plasma density and nonuniform $\left(B_{\min ,} z=30 \mathrm{~cm}=60 \mathrm{G}\right)$ magnetic field profiles at $p=1 \mathrm{mTorr}$. Note $z^{\prime}$ $=z+37.5 \mathrm{~cm}$.

The antenna input impedance value computed from our MAXEB code, which has the same magnetic field and density profiles incorporated in it as the experimental nonuniform configuration is, $0.63+j 14 \Omega$ as compared to the measurement of $0.92+j 11 \Omega$ at $13.56 \mathrm{MHz}$. This is for an input power of $1.1 \mathrm{~kW}$ with reflected power of $11 \mathrm{~W}$ at a pressure of 1 mTorr. For the uniform magnetic field case we obtain $0.85+j 10.4 \Omega$ in the experiment and $0.80+j 14 \Omega$ from the MAXEB code. The smaller radiation resistance obtained from the code is closer to the experimental one than previous one dimensional (1-D) comparisons in the literature. ${ }^{5}$ It should also be noted that the radiation resistance is more substantial for the nonuniform magnetic field case which corresponds with the experimental one. In addition, the code and experimental input resistance is quite close for a uniform magnetic field with the measured axial and radial plasma density profiles.

We compared the experimental measurements of the calibrated $B_{z}$ wave field with code predictions from the MAXEB code for the same coupled power levels. The variation of wave field in the experiment compared to the code for the uniform case is illustrated in Fig. 13. Figure 14 illustrates the result for the nonuniform case. The minima as well as the first and third peaks in the axial wave field agree quite well for the uniform case. The wave magnetic field peak near the left-hand side of the antenna for the nonuniform case is much larger than that predicted by the linear code but is more comparable as one moves away from the antenna region.

\section{DISCUSSION}

We have carried out wave, antenna impedance, and plasma measurements for a helicon source in nonuniform and uniform magnetic field configurations. Plasma density profiles as well as transverse and axial electron temperatures were measured. It was found that average densities and temperatures were somewhat higher for the nonuniform mag- 


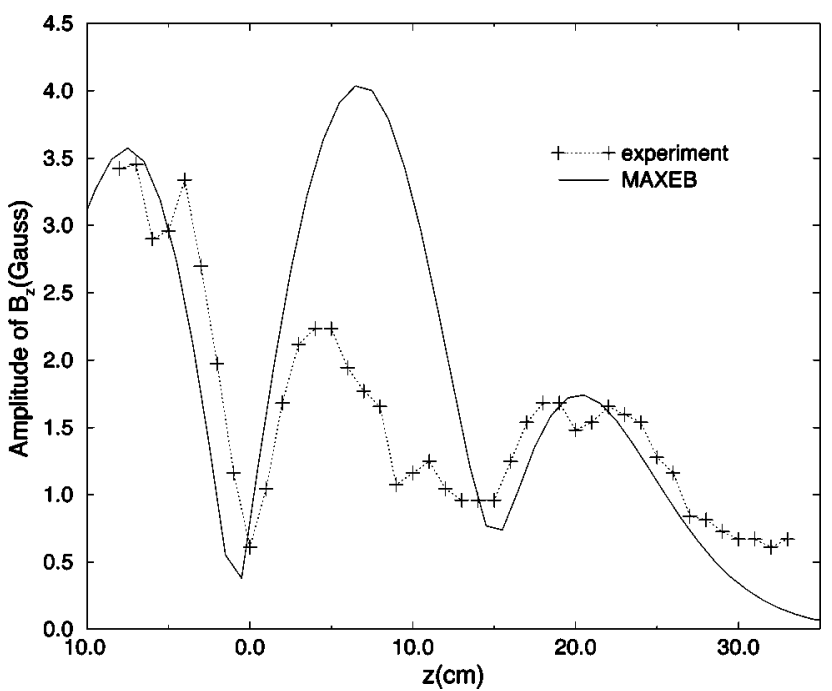

FIG. 13. $B_{z}$ wave field (at $r=0.63 \mathrm{~cm}$ ) for the plasma density shown in Fig. 2 in a uniform magnetic field. The $15-\mathrm{cm}$-long antenna is centered at $z=0$.

netic field case than for the same coupled power. In addition, a significant electron temperature anisotropy was measured for the nonuniform magnetic field case.

The strong axial density gradients near one side of the type-III antenna corresponding to the strong static magnetic field gradients there allow the antenna to operate essentially as a single-ended output plasma source. These plasma gradients act to reflect the helicon wave from one side of the source and at higher powers $(p>200 \mathrm{~W})$ produce a "blue core" plasma in argon indicative of high density and substantial fast electron creation. The "blue core" has a larger $(4.0$ vs. $3.0 \mathrm{~cm}$ ) diameter for the nonuniform configuration. It also remains fairly uniform axially as the magnetic field compresses and this is attributed to the strong wave fields $|E|=10-40 \mathrm{~V} / \mathrm{cm}$ and axially distributed wave heating.

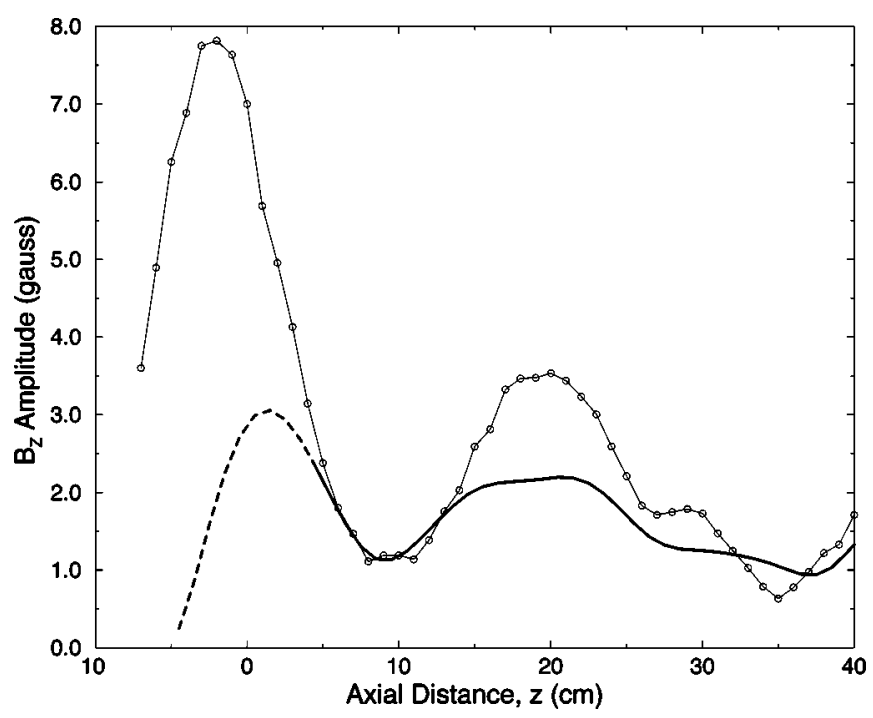

FIG. 14. $B_{z}$ wave field (at $r=0.63 \mathrm{~cm}$ ) for the measured plasma density and nonuniform magnetic field profiles. The $15-\mathrm{cm}-$ long antenna is centered at $z=0$.
It was found that the plasma characteristics were quite comparable whether or not the $\omega=\left|\omega_{c e}\right|$ cyclotron resonance was present for the nonuniform magnetic field case. The lack of a substantial cyclotron resonance heating process in the experiment is attributed to several effects. Strong wave reflection occurs in regions near the resonance zone. In this strong magnetic field gradient zone, the wave-electron phase correlation varies rapidly over a gyroperiod, thus reducing electron heating. In addition, the axial wave magnetic field amplitude is comparable to the static resonance field amplitude, thus strongly modifying the cyclotron resonance process. It was found that when nitrogen was the working gas for the source, operation with the nonuniform magnetic field configuration allowed the source to operate well at higher nitrogen pressures of 100 mTorr.

We carried out network analyzer measurements of the antenna input impedance for well-matched conditions and find that the nonuniform magnetic field has a slightly higher resistive component than the uniform case. The wave magnetic field and phase was measured for both the nonuniform and uniform magnetic field configurations. It was found that the nonuniform magnetic field configuration yields an intense axial wave magnetic field which decays rapidly away from the antenna region. Resonant electron velocities corresponding to the local phase velocity for the nonuniform magnetic field case range from $5 \mathrm{eV}$ on the left-hand side of the antenna region to $38 \mathrm{eV}$ well away from the antenna $(z$ $=20 \mathrm{~cm}$ ). This range of variation can efficiently dampen the wave and accelerate electrons from the background Maxwellian to higher energies needed to produce the "blue core.' The uniform magnetic field configuration has a higher range of local phase velocities corresponding to $25-40 \mathrm{eV}$. This wider range of local phase velocities down to thermal values near the antenna region for the nonuniform magnetic field case could give rise to more efficient heating and acceleration of electrons and production of higher densities and temperatures than the uniform case. As noted, the electron kinetic energy is double and the volume average beta is about four times that for the uniform magnetic field case for the same coupled power.

We also carried out 2-D simulations of the antenna coupling, input impedance, wave $B_{z}$ field, and collisional power absorption for the measured plasma and magnetic field profiles utilizing the linear MAXEB code. It was found that the antenna input impedances were comparable to the measured values and the wave $B_{z}$ field solution agreed well with the simulation for the uniform magnetic field case. For the nonuniform magnetic field case the simulation was comparable to measurements well away from the antenna region but did not indicate the strong peak near the left-hand side of the antenna. Substantial capacitive coupling, near field heating as noted by Borg et al., ${ }^{27}$ nonlinear wave-particle interactions and strong magnetic field gradients under the antenna could give rise to this difference. The code also indicates significant collisional plasma heating in the zone near the minimum magnetic field ( $B=60 \mathrm{G}$ ) for the nonuniform case.

It is anticipated that further improvements in helicon source operation are possible for nonuniform magnetic field configurations. The role of the full wave fields in heating and 
nonlinear electron wave heating and trapping processes for these sources is an important area for future research that will allow helicon sources to be utilized for a variety of applications.

\section{ACKNOWLEDGMENTS}

This work is primarily supported by Air Force Office of Scientific Research grant (Grant No. F49620-97-1-0262) in cooperation with the Defense Department Research and Engineering Air Plasma Ramparts Multi-University Research Initiative program. It is also supported in part by National Science Foundation Grant No. ECS-9632377 and by the University of Wisconsin-Madison. We also thank Mr. Guowen Ding for discussions regarding Langmuir probe data interpretation.

${ }^{1}$ R. W. Boswell, Plasma Phys. Controlled Fusion 26, 1147 (1984).

${ }^{2}$ A. Komori, T. Shoji, K. Miyamoto, J. Kawai, and K. Kawai, Phys. Fluids B 3, 893 (1991).

${ }^{3}$ T. Shoji, Y. Sakawa, S. Nakazawa, K. Kadota, and T. Sato, Plasma Sources Sci. Technol. 2, 5 (1993).

${ }^{4}$ F. F. Chen, Plasma Phys. Controlled Fusion 33, 339 (1991).

${ }^{5}$ A. W. Molvik, A. R. Ellingboe, and T. D. Ronglien, Phys. Rev. Lett. 79, 233 (1997).

${ }^{6}$ A. R. Ellingboe, R. W. Boswell, J. P. Booth, and N. Sadeghi, Phys. Plasmas 2, 1807 (1995).
${ }^{7}$ A. W. Degeling, C. O. Jung, R. W. Boswell, and A. R. Ellingboe, Phys. Plasmas 3, 2788 (1996).

${ }^{8}$ R. T. S. Chen and N. Hershkowitz, Phys. Rev. Lett. 80, 4677 (1998).

${ }^{9}$ P. A. Keiter, E. E. Scime, and M. M. Balkey, Phys. Plasmas 4, 2741 (1997).

${ }^{10}$ M. A. Lieberman and A. J. Lichtenberg, Principles of Plasma Discharges And Materials Processing (Wiley, New York, 1994).

${ }^{11}$ P. K. Loewenhardt, R. D. Blackwell, R. W. Boswell, G. D. Conway, and S. M. Hamberger, Phys. Rev. Lett. 67, 2792 (1991).

${ }^{12}$ P. Zhu and R. W. Boswell, Phys. Rev. Lett. 63, 2805 (1989).

${ }^{13} \mathrm{~J}$. Gilland (private communication).

${ }^{14}$ R. W. Boswell and F. F. Chen, IEEE Trans. Plasma Sci. 25, 1229 (1997).

${ }^{15}$ F. F. Chen and G. Chevalier, J. Vac. Sci. Technol. A 10, 1389 (1992).

${ }^{16}$ F. F. Chen and R. W. Boswell, IEEE Trans. Plasma Sci. 25, 1245 (1997).

${ }^{17}$ J. Gilland, R. Breun, and N. Hershkowitz, Plasma Sources Sci. Technol. 7, 416 (1998).

${ }^{18}$ L. A. Berry and J. C. Whitson (private communication).

${ }^{19}$ Y. Mouzouris and J. E. Scharer, Phys. Plasmas 5, 4253 (1998).

${ }^{20}$ H. Aikawa, J. Phys. Soc. Jpn. 40, 1741 (1976).

${ }^{21}$ H. Amemiya, H. Oyama, and Y. Sakamoto, J. Phys. Soc. Jpn. 56, 2401 (1987)

${ }^{22}$ G. Himmel, M. Holtkamp, and H. Schluter, Plasma Phys. Controlled Fusion 34, 923 (1992).

${ }^{23}$ M. Tichy, P. Kudrna, J. F. Behnke, C. Csambal, and S. Klagge, J. Phys. IV 7, 397 (1997).

${ }^{24}$ I. D. Sudit and F. F. Chen, Plasma Sources Sci. Technol. 5, 43 (1996).

${ }^{25}$ M. Light and F. F. Chen, Phys. Plasmas 2, 1084 (1995).

${ }^{26}$ A. H. Sarkissian, E. Charette, B. C. Gregory, and D. Larosa, Plasma Sources Sci. Technol. 5, 760 (1996).

${ }^{27}$ G. Borg, J. Bright, and I. V. Kamenski, Plasma Phys. Control. Fusion 40, 987 (1998). 